\title{
The Holder continuity of the solutions to quasi-linear system of elliptic partial differential equations with singular coefficients
}

\author{
Mykola I. Yaremenko \\ National Technical University, Ukraine \\ Received: October 2020. Accepted : October 2021
}

\begin{abstract}
This article establishes the Holder continuity of the solutions to a quasi-linear system of elliptic partial differential equations with singular coefficients under the assumption of its form-boundary.
\end{abstract}

Keywords: Holder continuity, partial differential equation, singular coefficients, Sobolev space.

Subject classification: $35 J 15$, 35J60, 35K62. 


\section{Introduction}

This article is dedicated to the Holder regularity conditions for the solutions to the quasi-linear system of elliptic partial differential equations with singular slow-growing coefficients [1-6]. In our previous works was shown that such a system has the solutions in Sobolev space [1]; in the present article, the Holder properties of these solutions are studied [47-49], [7-10, 36, 37]. The main goal of this article to establish conditions under which the solutions to a quasi-linear system of elliptic partial differential equations belong to the functional Holder space.

Let us consider the quasi-linear system of elliptic partial differential equations

$(1 \lambda \vec{u} l)^{k}-\sum_{i, j=1, \ldots, l} \frac{\partial}{\partial x_{i}}\left(a_{i j}(x, \vec{u}) \frac{\partial}{\partial x_{j}} u^{k}\right)+b^{k}(x, \vec{u}, \nabla \vec{u})=f^{k}, \quad k=1, \ldots, N$

where $\lambda>0$ is a real number; the $\vec{u}$ is an unknown vector-function of vector-argument $x \in R^{l}, \quad l>2$ and $\vec{f}=f(x)$ is given vector-function $f \in L^{p} \cap L^{\infty}$. The $\vec{b}(x, u, \nabla u)$ is given vector-function. $1 . b(x, y, z)$ is a real measurable function of its arguments and $b \in L_{l o c}^{1}\left(R^{l}\right) ; 2$. Function $\vec{b}(x, y, z)$ almost everywhere satisfies an inequality

$$
|\vec{b}(x, \vec{u}, \nabla \vec{u})| \leq \mu_{1}(x)|\nabla \vec{u}|+\mu_{2}(x)|\vec{u}|+\mu_{3}(x)
$$

where $\mu_{1}^{2} \in P K_{\beta}(A), \mu_{2} \in P K_{\beta}(A), \mu_{3} \in L^{p}\left(R^{l}\right) ; 3$. Growth of the function $\vec{b}(x, y, z)$ almost everywhere satisfies a condition

$$
|\vec{b}(x, \vec{u}, \nabla \vec{u})-\vec{b}(x, \vec{v}, \nabla \vec{v})| \leq \mu_{4}(x)|\nabla(\vec{u}-\vec{v})|+\mu_{5}(x)|\vec{u}-\vec{v}|,
$$

where $\mu_{4}^{2} \in P K_{\beta}(A), \mu_{5} \in P K_{\beta}(A)$.

The $a_{i j}(x)$ is a measurable matrix of $l \times l$ size and satisfies the condition $\exists \nu, \mu: \quad 0<\nu<\mu<\infty$ such that

$$
\nu \sum_{i=1}^{l} \xi_{i}^{2} \leq \sum_{i j=1, \ldots, l} a_{i j} \xi_{i} \xi_{j} \leq \sum_{i=1}^{l} \xi_{i}^{2} \quad \forall \xi \in R^{l}
$$

The functional class of form-bounded functions $P K_{\beta}$ can be defined as

$$
P K_{\beta}(A)=\left\{f \in L_{l o c}^{1}\left(R^{l}, d^{l} x\right): \quad\left|\left\langle f|h|^{2}\right\rangle\right| \leq \beta\left\langle A^{\frac{1}{2}} h, A^{\frac{1}{2}} h\right\rangle+c(\beta)\|h\|_{2}^{2}\right\},
$$


where a function $h \in D\left(A^{\frac{1}{2}}\right)$ and a number $\beta>0$ is a form-boundary and constantc $(\beta) \in R^{1}[6]$.

Let us denote

$$
\begin{aligned}
& \|\vec{u}\|_{L^{p}\left(R^{l}\right)}=\left\langle\sum_{i=1, \ldots, N}\left|u^{i}\right|^{p}\right\rangle^{\frac{1}{p}}=\left(\sum_{i=1, \ldots, N}\left\langle\left|u^{i}\right|^{p}\right\rangle\right)^{\frac{1}{p}}, \\
& \langle\vec{u}, \vec{v}\rangle=\sum_{i=1, \ldots, N}\left\langle u^{i}, v^{i}\right\rangle \quad \forall u \in L^{p}\left(R^{l}\right) \forall v \in L^{q}\left(R^{l}\right),
\end{aligned}
$$

then we have equality

$$
\|\vec{u}\|_{L^{p}\left(R^{l}\right)}^{p-1}=\left\langle\sum_{i=1, \ldots, N}\left|u^{i}\right|^{p}\right\rangle^{\frac{p-1}{p}}=\left\langle\sum_{i=1, \ldots, N}\left(\left|u^{i}\right|^{\frac{p}{q}}\right)^{q}\right\rangle^{\frac{1}{q}}=\left\||\vec{u}|^{p-1}\right\|_{L^{q}\left(R^{l}\right)} .
$$

Next, we denote

$$
|\nabla \vec{u}|^{p}=\sum_{i=1, \ldots, N} \sum_{k=1, \ldots l}\left|\frac{\partial}{\partial x_{k}} u^{i}\right|^{p}
$$

and

$$
\|u\|_{p}^{p}=\left\langle\sum_{i=1}^{N} u^{i} u^{i}\left|u^{i}\right|^{p-2}\right\rangle \equiv \sum_{i=1}^{N}\left\langle u^{i} u^{i}|u|^{p-2}\right\rangle .
$$

Definition (of weak solution). A vector-function $\vec{u} \in W_{1}^{p}\left(R^{l}, d^{l} x\right)$ is called a weak solution to a quasilinear system of elliptic partial differential equations if the integral identity

$$
\lambda\langle\vec{u}, \vec{\nu}\rangle+\left\langle\sum_{i, j=1, \ldots, l} a_{i j} \frac{\partial}{\partial x_{j}} \vec{u}, \frac{\partial}{\partial x_{i}} \vec{\nu}\right\rangle+\langle\vec{b}, \vec{\nu}\rangle=\langle\vec{f}, \vec{\nu}\rangle
$$

is valid for all vector-functions $v \in W_{1,0}^{q}\left(R^{l}, d^{l} x\right)$.

The main result of this article can be formulated follows.

Theorem 1. The weak solution $\vec{u} \in W_{1}^{p}\left(R^{l}, d^{l} x\right)$ to the quasilinear system (1) under the assumptions 1-4 belongs to Holder space of continuous functions. 


\section{The estimation of the main part of the elliptic differential operator}

Let us consider a simpler elliptic system

$$
\frac{\partial}{\partial x_{i}}\left(a_{i j}(x) \frac{\partial}{\partial x_{j}} \vec{u}\right)=0,
$$

let us compose the integral identity as

$$
\left\langle\sum_{i, j=1, \ldots, l} a_{i j} \frac{\partial}{\partial x_{j}} \vec{u}, \frac{\partial}{\partial x_{i}} \vec{v}\right\rangle=0
$$

where $\vec{v} \in W_{1,0}^{2}\left(R^{l}, d^{l} x\right)$ and $\vec{u} \in W_{1}^{2}\left(R^{l}, d^{l} x\right)$ under the ellipticity condition and the condition vrai max $|\vec{u}|<\infty$.

Let us assume that function $\vec{u}(x)$ measurable in a ball $K_{\rho_{0}}$ and there are $N_{1}$ functions $\vec{w}^{1}(x), \ldots, \vec{w}^{N_{1}}(x)$ such that for the arbitrary ball $K_{b \rho}$, concentric with $K_{\rho_{0}}$, there is at least one function $\vec{w}^{r}(x)$ such that

$$
\operatorname{osc}\left\{\left|\vec{w}^{r}\right|, \Omega_{b \rho}\right\} \geq \delta_{1} \max \operatorname{osc}\left\{|\vec{u}|, \Omega_{b \rho}\right\},
$$

for the function $\vec{u}(x)$, we are obtaining that holds at least one of the following inequalities

$$
\operatorname{osc}\left\{\left|\vec{w}^{r}\right|, \Omega_{\rho}\right\} \leq c_{1} \rho^{\delta},
$$

Or

$$
\operatorname{osc}\left\{\left|\vec{w}^{r}\right|, \Omega_{\rho}\right\} \leq \vartheta \operatorname{osc}\left\{\left|\vec{w}^{r}\right|, \Omega_{b \rho}\right\},
$$

where the balls $K_{\rho_{0}}, K_{\rho}$ and $K_{b \rho}$ have the same center, and constant $b$ is a fix; others satisfy the following conditions $b \rho \leq \rho_{0}, b>1, c_{1} \leq 1, \delta \leq$ $1, \vartheta<1 \Omega_{\rho}=\Omega \cap K_{\rho}$.

Then for $\rho \leq \rho_{0}$ there is an estimation

$$
\operatorname{osc}\left\{u, \Omega_{\rho}\right\} \leq A\left(\frac{\rho}{\rho_{0}}\right)^{\alpha},
$$

where we denote

$$
\alpha=\frac{1}{N_{1}} \min \left\{-\log _{b} \vartheta, \delta\right\}, c=\frac{b^{\alpha\left(N_{1}+1\right)}}{\delta_{1}} \max \left\{b^{\alpha N_{1}} \max _{i=1, \ldots, N_{1}} \operatorname{osc}\left\{\left|\vec{w}^{i}\right|, \Omega_{\rho_{0}}\right\}, c_{1} \rho_{0}^{\delta}\right\}
$$
and $u=|\vec{u}|$.

To assert that the function $\vec{u}$ belongs to Holder space enough to show that

$$
\operatorname{osc}\left\{|\vec{u}|, K_{R}\right\} \leq \vartheta \operatorname{osc}\left\{|\vec{u}|, K_{2 R}\right\} .
$$


For a positive number $\varepsilon>0$, let us consider the function $\psi(\vec{u})=$ $-\ln 2(1-|\vec{u}|+\varepsilon)$, presuppose that in the ball $K_{R}$ holds the estimation $-\ln 2(1-|\vec{u}|+\varepsilon)<L$, then we obtain that $\frac{\exp (-L)}{2}<1-|\vec{u}|+\varepsilon$, or $|\vec{u}(x)|<1-\frac{\exp (-L)}{2}+\varepsilon$ if we put $\vartheta=1-\frac{\exp (-L)}{2}+\varepsilon$ and the number $\varepsilon>0$ converges to zero we obtain that $\operatorname{osc}\left\{\vec{u}, K_{R}\right\} \leq \vartheta \operatorname{osc}\left\{\vec{u}, K_{2 R}\right\}$. So, we have to show that the function $w(x)=\psi(\vec{u})(x)=-\ln 2(1-|\vec{u}(x)|+\varepsilon)$ is bounded.

It can be assumed that the oscillation of function $\vec{u}(x)$ in the ball $K_{2 R}$ equals one, that is $0 \leq \vec{u}(x) \leq 1$, then one of the properties is always executed:

$$
\begin{gathered}
\text { mes }\left\{x \in K_{R},|\vec{u}(x)| \leq \frac{1}{2}\right\} \geq \frac{1}{2}{\text { mes } K_{R},}, \\
\text { mes }\left\{x \in K_{R}, 1-|\vec{u}(x)| \leq \frac{1}{2}\right\} \geq \frac{1}{2}{\text { mes } K_{R},},
\end{gathered}
$$

if holds the first property we consider the function $\vec{u}(x)$, if the second is true we consider $1-\vec{u}(x)$. Assume that the first property executes then for arbitrary function $\vec{v} \in W_{1,0}^{2}\left(K_{2 R}\right)$, we have

$$
\left\langle\sum_{i, j=1, \ldots, l} a_{i j} \frac{\partial}{\partial x_{j}} \vec{u}, \frac{\partial}{\partial x_{i}} \vec{v}\right\rangle_{K_{2 R}}=0 .
$$

To show that the function $w(x)=\psi(\vec{u})(x)=-\ln 2(1-|\vec{u}(x)|+\varepsilon)$ is bounded above, we denote

$$
\vec{v}(x)=\frac{\vec{\xi}(x)}{1-|\vec{u}(x)|+\varepsilon}=\psi^{\prime}(\vec{u})(x) \vec{\xi}(x)
$$

since

we are obtaining

$$
\frac{\partial}{\partial x_{i}} \vec{v}=\psi^{\prime \prime} \vec{\xi} \frac{\partial}{\partial x_{i}}|\vec{u}|+\psi^{\prime} \frac{\partial}{\partial x_{i}} \vec{\xi}
$$

$$
\begin{gathered}
\left\langle\sum_{i, j=1, \ldots, l} a_{i j} \frac{\partial}{\partial x_{j}} \vec{u}, \psi^{\prime \prime} \vec{\xi} \frac{\partial}{\partial x_{i}}|\vec{u}|\right\rangle_{K_{2 R}}+\left\langle\sum_{i, j=1, \ldots, l} a_{i j} \frac{\partial}{\partial x_{j}} \vec{u}, \psi^{\prime} \frac{\partial}{\partial x_{i}} \vec{\xi}\right\rangle_{K_{2 R}}=0 \\
\left\langle\sum_{i, j=1, \ldots, l} a_{i j} \frac{\partial}{\partial x_{j}} \vec{w}, \vec{\xi} \frac{\partial}{\partial x_{i}}|\vec{w}|\right\rangle_{K_{2 R}}+\left\langle\sum_{i, j=1, \ldots, l} a_{i j} \frac{\partial}{\partial x_{j}} \vec{w}, \frac{\partial}{\partial x_{i}} \vec{\xi}\right\rangle_{K_{2 R}}=0 .
\end{gathered}
$$

Denote function $\xi=\varphi^{2}\left(\frac{\left|x-x_{0}\right|}{R}\right)$, where the function $\varphi(t)$ equals one if $\frac{\left|x-x_{0}\right|}{R} \in\left[0, \frac{3}{2}\right]$ and if argument equals two $\varphi(t)$ linearly decreases to zero 
$\left|x-x_{0}\right|=2 R$. Applying elliptic condition $\nu \sum_{i=1}^{l} \xi_{i}^{2} \leq \sum_{i j=1, \ldots, l} a_{i j}(x) \xi_{i} \xi_{j} \leq$ $\mu \sum_{i=1}^{l} \xi_{i}^{2} \quad \forall \xi \in R^{l}$, we obtain

$$
\left\langle|\nabla \vec{w}|^{2}\right\rangle_{K_{\frac{3}{2} R}} \leq \frac{16 \mu}{\nu R^{2}} \operatorname{mes} K_{2 R}
$$

Next step, we will apply Moser's idea: that from the boundedness of the convex function

$$
w(x)=\psi(\vec{u})(x)=-\ln 2(1-|\vec{u}(x)|+\varepsilon)
$$

follows that the function $\vec{u}$ is Holder continuous.

Since $0 \leq \vec{u}(x) \leq 1$ and

$$
w(x)=\psi(\vec{u})(x)=-\ln 2(1-|\vec{u}(x)|+\varepsilon)
$$

we have

$$
\inf w(x)=-\ln 2(1+\varepsilon) .
$$

The value $\left\langle|w|^{2}\right\rangle_{K_{\frac{3}{2} R}}$ can be estimated by applying the De Giorgi method or Nash estimation, we will use the De Giorgi lemma [23, 24].

Let vector $\vec{u} \in W_{1}^{p}(\Omega)$, for all positive number $k$, we denote by $A_{k}$ the set $A_{k} \equiv\{x \in \Omega:|\vec{u}(x)|>k\}$ and the sets $A_{k}^{0} \equiv\{x \in \Omega:|\vec{u}(x)|=k\}$, $A_{k, \rho} \equiv\left\{x \in K_{\rho}:|\vec{u}(x)|>k\right\}$ and function $u_{k}(x)=\max (|\vec{u}(x)|-k, 0)$. Form definition we deduce the following properties:

$$
\begin{gathered}
1 . A_{k}=\bigcup_{\varepsilon>0} A_{k+\varepsilon} \\
\text { 2.mes }\left(A_{k} \backslash A_{k+\varepsilon}\right) \stackrel{\varepsilon \rightarrow 0}{\longrightarrow} 0 \\
\text { 3.mes }\left(A_{k-\varepsilon} \backslash A_{k} \bigcup A_{k}^{0}\right) \stackrel{\varepsilon \rightarrow 0}{\longrightarrow} 0 \\
4 . u_{k} \in W_{1}^{p}(\Omega) .
\end{gathered}
$$

Lemma 1. Let $\vec{u} \in W_{1}^{2}\left(K_{\rho}\right)$ and $A$ is an arbitrary subset of a set $K_{\rho}$, denote set $A_{0} \equiv\left\{x \in K_{\rho}:|\vec{u}(x)|=0\right\}$ and positive number $\beta>0$ that only depended on the dimension of space then for all $k \geq n$ the following inequalities hold

$$
\langle|\vec{u}|\rangle_{A} \leq \beta \frac{\rho^{l}}{m e s A_{0}}(m e s A)^{\frac{1}{l}}\langle|\nabla \vec{u}|\rangle_{K_{\rho}},
$$




$$
\begin{gathered}
(n-k)\left(\operatorname{mes}_{n, \rho}\right)^{1-\frac{1}{l}} \leq \beta \frac{\rho^{l}}{\operatorname{mes}\left(K_{\rho} \backslash A_{n, \rho}\right) A_{0}}\langle|\nabla \vec{u}|\rangle_{A_{k, \rho} \backslash A_{n, \rho}}, \\
\left\langle\left|\nabla\left(\left|\vec{u}_{k}\right|^{m}\right)\right|\right\rangle_{K_{\rho}} \leq m\left\langle|\nabla \vec{u}|^{m}\right\rangle_{A_{k, \rho}}^{\frac{1}{m}}\left\langle|| \vec{u}|-k|^{m}\right\rangle_{A_{k, \rho}}^{1-\frac{1}{m}} .
\end{gathered}
$$

There is a constant that $c$ that depends only on the dimension of the space and the ellipticity of the matrix, such that

$$
\left\langle|\nabla w|^{2}\right\rangle_{K_{\frac{3}{2} R}} \leq c R^{l}
$$

Let us denote

$$
\xi(x)=\varphi^{2}(x) \max (w(x)-k, 0) \quad \forall k
$$

where the function $\varphi$ is a cutoff function for the ball $K_{\rho}, \quad \rho \in\left[R, \frac{3}{2} R\right]$ then we obtain estimation (here we denote $A_{k, \rho} \equiv\left\{x \in K_{\rho}: w(x)>k\right\}$ )

$$
\left\langle\sum_{i, j=1, \ldots, l} a_{i j} \frac{\partial}{\partial x_{j}} w, \varphi^{2} \frac{\partial}{\partial x_{i}} w\right\rangle_{A_{k, \rho}}+\left\langle\sum_{i, j=1, \ldots, l} a_{i j} \frac{\partial}{\partial x_{j}} w, 2 \varphi(w-k) \frac{\partial}{\partial x_{i}} \varphi\right\rangle_{A_{k, \rho}} \leq 0
$$

that is

$$
\left\langle|\nabla w|^{2} \varphi^{2}\right\rangle_{A_{k, \rho}} \leq !\left\langle(w-k)^{2}|\nabla \varphi|^{2}\right\rangle_{A_{k, \rho}},
$$

thus, we have obtained that there is a constant $M$ that depends only on the ellipticity constants and dimension $l$ of the space such that holds inequality

$$
\operatorname{vrai} \max _{K_{R}} w(x)<M
$$

so, the function-solution $u$ belongs to the Holder's functional class.

Lemma 2. Let $\vec{u}(x)$ is a given measurable in a ball $K_{1}$ function and balls $K_{1}, K_{\rho}$ and $K_{\rho-\sigma \rho}$ with a common center, and constants $1>\sigma_{0}>0, \gamma>$ $0, \alpha>0, \varepsilon>0$, and $\varepsilon \leq \frac{m}{l}, m \leq \alpha<\varepsilon m+m$, and for arbitrary natural number $k>k_{0}$ holds the inequality

$$
\left\langle|\nabla \vec{u}|^{m}\right\rangle_{A_{k, \rho-\sigma \rho}} \leq \gamma \sigma^{-m}\left\langle(|u|-k)^{m}\right\rangle_{A_{k, \rho}}+\gamma k^{\alpha}\left(\text { mes } A_{k, \rho}\right)^{1-\frac{m}{l}+\varepsilon} .
$$

Then in the ball $K_{1-\sigma}$, the value vrai $\max _{K_{1-\sigma}}|\vec{u}(x)|$ can be estimated by a constant that only depends on $\sigma_{0}, \gamma, \alpha, \varepsilon, k_{0}, m, l$ and magnitude $a=\left\langle|| \vec{u}(\cdot)|-k|^{m}\right\rangle_{A_{k_{0}, 1}}$. 
Proof. Let $\tilde{k}>k_{0}$ be the natural number and consider the sequence $K_{\rho_{i}}$ of balls having a common center and with radii $\rho_{i}=1-\sigma_{0}+\frac{\sigma_{0}}{2^{i}}, i=$ $0,1,2, \ldots$. , and the sequence of the planes $k_{i}=2 \tilde{k}-\frac{\tilde{k}}{2^{i}}, i=0,1,2, \ldots$. Let us denote $\xi(t)$ the continuous differentiable non-increase function of the argument $t \in(-\infty, \infty)$, which equals one when $t \leq \sigma_{0}$ and zero when $t \geq \frac{3}{2} \sigma_{0}$ next we denote the sequence of functions

$$
\xi_{i}(t)=\xi\left(2^{i+1}\left(|x|-1+\sigma_{0}\right)\right), i=0,1,2, \ldots
$$

and sequence of numbers

$$
J_{i}=\left\langle\left(u-k_{i}\right)^{m}\right\rangle_{A_{k_{i}, \rho_{i}}}, i=0,1,2, \ldots
$$

We estimate

$$
J_{i+1} \leq\left\langle\left(u-k_{i+1}\right)^{m} \xi_{i}^{m}\right\rangle_{k_{i+1}, \frac{\rho_{i}+\rho_{i+1}}{2}}, i=0,1,2, \ldots
$$

and applying Holder estimation, we are obtaining recurrent inequality

$$
\begin{aligned}
& J_{i+1} \leq\left\langle\left(u-k_{i+1}\right)^{m} \xi_{i}^{m}\right\rangle_{A_{k_{i+1}, \frac{\rho_{i}+\rho_{i+1}}{2}}} \leq \\
& \leq C\left(m e s A_{k_{i+1}, \frac{\rho_{i}+\rho_{i+1}}{2}}\right)^{\frac{m_{l}}{l}}\left\langle|\nabla u|^{m}\right\rangle_{A_{k_{i+1},}, \frac{\rho_{i}+\rho_{i+1}}{2}}+ \\
& +C\left(m e s A_{k_{i+1}, \frac{\rho_{i}+\rho_{i+1}}{2}}\right)^{\frac{m}{l}} \max _{t \in\left[\sigma_{0}, \frac{3}{2} \sigma_{0}\right]}\left|\xi^{\prime}(t)\right|^{m} 2^{m i}\left\langle\left(u-k_{i}\right)^{m}\right\rangle_{A_{k_{i}, \rho_{i}}}= \\
& =C\left(m e s A_{k_{i+1}, \frac{\rho_{i}+\rho_{i+1}}{2}}\right)^{\frac{m}{l}}\left\langle|\nabla u|^{m}\right\rangle_{A_{k_{i+1}}, \frac{\rho_{i}+\rho_{i+1}}{2}}+ \\
& +C\left(m e s A_{k_{i+1}, \frac{\rho_{i}+\rho_{i+1}}{2}}\right)^{\frac{m}{l}} \max _{t \in\left[\sigma_{0}, \frac{3}{2} \sigma_{0}\right]}\left|\xi^{\prime}(t)\right|^{m} 2^{m i} J_{i}, i=0,1,2, \ldots
\end{aligned}
$$

We put $k=k_{i+1}, \rho=\rho_{i}, \rho-\sigma \rho=\frac{\rho_{i}+\rho_{i+1}}{2}$, then

$$
\begin{aligned}
& \left\langle|\nabla u|^{m}\right\rangle_{A_{i+1}, \frac{\rho_{i}+\rho_{i+1}}{2}} \leq \gamma 2^{i m+3 m}\left\langle\left|u-k_{i+1}\right|^{m}\right\rangle_{A_{k_{i+1}, \rho_{i}}}+ \\
& +\gamma k_{i+1}^{\alpha}\left(m e s A_{k_{i+1}, \rho_{i}}\right)^{1-\frac{m}{l}+\varepsilon} \leq \\
& \leq \gamma\left(2^{3 m}+2^{\alpha}\right) 2^{m i} J_{i}+\gamma\left(2^{3 m}+2^{\alpha}\right) \tilde{k}^{\alpha}\left(m e s A_{k_{i+1}, \rho_{i}}\right)^{1-\frac{m}{l}+\varepsilon} .
\end{aligned}
$$

Further, we assess

$$
J_{i} \geq\left\langle\left|u-k_{i}\right|^{m}\right\rangle_{A_{k_{i+1}, \rho_{i}}} \geq\left(k_{i+1}-k_{i}\right)^{m}\left(\operatorname{mes} A_{k_{i+1}, \rho_{i}}\right)=2^{-m(i+1) \tilde{k}^{m}} m e s A_{k_{i+1}, \rho_{i}} .
$$


Then, when $\frac{m^{2}}{l} \geq m+m \varepsilon-\alpha>0$, we have

$$
J_{i+1} \leq C_{1} 2^{i\left(m+\frac{m^{2}}{l}\right)}\left(\tilde{k}^{-\frac{m^{2}}{l}} J_{i}^{1+\frac{m}{l}}+\tilde{k}^{-m-m \varepsilon+\alpha} J_{i}^{1+\varepsilon}\right) .
$$

However, the estimations

$$
J_{i}=\left\langle\left(u-k_{i}\right)^{m}\right\rangle_{A_{k_{i}, \rho_{i}}} \leq\left\langle(u-\tilde{k})^{m}\right\rangle_{A_{k_{0}, 1}}, i=0,1,2, \ldots
$$

are holding and so for $\tilde{k} \geq 1$ we are obtaining the recurrent inequalities $J_{i+1} \leq C_{1}\left(1+\left\langle(u-\tilde{k})^{m}\right\rangle_{A_{k_{0}, 1}}^{\frac{m}{l}-\varepsilon}\right) 2^{i\left(m+\frac{m^{2}}{l}\right)} \tilde{k}^{-m-m \varepsilon+\alpha} J_{i}^{1+\varepsilon}, i=0,1,2, \ldots .$.

Let us choose the value $\tilde{k} \geq 1$ such that the inequality

$$
\begin{gathered}
\tilde{k} \geq \max \left(k_{0}, 1,\left(C_{1} 1+C_{1}\left\langle(u-\tilde{k})^{m}\right\rangle_{A_{k_{0}, 1}}^{\frac{m}{l}-\varepsilon}\right)^{\frac{1}{m+m \varepsilon-\alpha}}\right. \\
\left.2^{\left(m+\frac{m^{2}}{l}\right) \frac{1}{\varepsilon(m+m \varepsilon-\alpha)(1+\varepsilon)}}\left\langle(u-\tilde{k})^{m}\right\rangle_{A_{k_{0}, 1}}^{\frac{\varepsilon}{m+m \varepsilon-\alpha}}\right)
\end{gathered}
$$

holds. So, we have

$$
\begin{aligned}
& J_{1} \leq\left(C_{1} 1+C_{1}\left\langle(u-\tilde{k})^{m}\right\rangle_{A_{k_{0}, 1}}^{\frac{m}{l}-\varepsilon}\right) \tilde{k}^{-m-m \varepsilon+\alpha}\left\langle(u-\tilde{k})^{m}\right\rangle_{A_{k_{0}, 1}}^{1+\varepsilon} \leq \\
& \leq \tilde{k}^{\frac{m+m \varepsilon-\alpha}{\varepsilon}}\left(C_{1} 1+C_{1}\left\langle(u-\tilde{k})^{m}\right\rangle_{A_{k_{0}, 1}}^{\frac{m}{l}-\varepsilon}\right)^{-\frac{1}{\varepsilon}} 2^{-\left(m+\frac{m^{2}}{l}\right) \frac{1}{\varepsilon^{2}}}
\end{aligned}
$$

applying the recursivity of the last estimation we obtain

$$
\begin{aligned}
& J_{i+1} \leq \text { Const } 2^{-\left(m+\frac{m^{2}}{l}\right) \frac{i}{\varepsilon}}, i=0,1,2, \ldots . \\
& J_{i+1} \stackrel{i \rightarrow \infty}{\longrightarrow} 0 .
\end{aligned}
$$

Thus, we have obtained vrai $\max _{K_{1-\sigma_{0}}}|u(x)|=2 \tilde{k}$, the lemma 2 has been proven.

Lemma 3. Let function $\vec{u} \in W_{1}^{1}(\Omega), \quad l>2$ and $B(r)$ is a ball radius $r$. Then there is an estimation

$$
\operatorname{mes}(\Theta)\langle|\vec{u}|\rangle_{\Xi} \leq \beta r^{l}(\operatorname{mes}(\Xi))^{\frac{1}{l}}\langle|\nabla \vec{u}(\cdot)|\rangle_{B(r)},
$$


here $\Theta$ is a set of points of the ball $B(r)$ such that $\vec{u}(x)=0$, and constant $\beta$ is a function of the dimension of Euclid space.

Proof. For almost every $x \in B(r)$ and $y \in \Xi$, there is a representation

$$
\vec{u}(y)-\vec{u}(x)=\int_{0}^{|x-y|} \frac{\partial \vec{u}(x+\omega \rho)}{\partial \rho} d \rho
$$

where $(\rho, \omega)$ are spherical coordinates. Next, we integrate this with respect to $y \in \Xi$ and obtain a iquality

$$
-\vec{u}(x) \operatorname{mes}(\Theta)=\left\langle\int_{0}^{|x-y|} \frac{\partial \vec{u}(x+\omega \rho)}{\partial \rho} d \rho\right\rangle_{\Theta} .
$$

We can estimate

$$
\begin{aligned}
& \left\langle\int_{0}^{|x-y|} \frac{\partial \vec{u}(x+\omega \rho)}{\partial \rho} d \rho\right\rangle_{\Theta} \leq \\
& \leq \int_{B(r)}|x-y|^{l-1} d|x-y| \int_{0}^{|x-y|} \frac{\partial \vec{u}(x+\omega \rho)}{\partial \rho} d \rho \leq \\
& \leq \int_{0}^{2 r}|x-y|^{l-1} d|x-y|\left\langle\frac{|\nabla \vec{u}(\cdot)|}{|\cdot-\xi|^{l-1}}\right\rangle_{B(r)}=\frac{(2 r)^{l}}{l}\left\langle\frac{|\nabla \vec{u}(\cdot)|}{|\cdot-\xi|^{l-1}}\right\rangle_{B(r)}
\end{aligned}
$$

so, we have an inequality

$$
|\vec{u}(x)| \operatorname{mes}(\Theta) \leq \frac{(2 r)^{l}}{l}\left\langle\frac{|\nabla \vec{u}(\cdot)|}{|\cdot-\xi|^{l-1}}\right\rangle_{B(r)} .
$$

We integrate over $\Xi$

$$
\langle|\vec{u}|\rangle_{\Theta} \text { mes }(\Theta) \leq \frac{(2 r)^{l}}{l} \int_{B(r)}|\nabla \vec{u}(y)| d y \int_{\Xi} \frac{d \xi}{|y-\xi|^{l-1}} .
$$

It is easy to see that

$$
\int_{|y-\xi| \leq \varepsilon} \frac{d \xi}{|y-\xi|^{l-1}}=\varepsilon \cdot \operatorname{mes}(S)
$$

and

$$
\int_{|y-\xi| \geq \varepsilon} \frac{d \xi}{|y-\xi|^{l-1}} \leq \varepsilon^{1-l} \cdot \operatorname{mes}(\Xi)
$$

so, we obtain an estimation

$$
\int_{\Xi} \frac{d \xi}{|y-\xi|^{l-1}} \leq \varepsilon \cdot \operatorname{mes}(S)+\varepsilon^{1-l} \cdot \operatorname{mes}(\Xi) .
$$

Lemma 3 is proven. 


\section{Quasilinear system of elliptic partial differential equations with nonlinear perturbation}

Let us consider a more general case of a quasilinear system of elliptic partial differential equations with nonlinear perturbation $\vec{b}$

$$
\lambda \vec{u}-\frac{\partial}{\partial x_{i}}\left(a_{i j}(x, \vec{u}) \frac{\partial}{\partial x_{j}} \vec{u}\right)+\vec{b}(x, \vec{u}, \nabla \vec{u})=0,
$$

The investigation will be carried out according to the scheme: we study the solution $\vec{u} \in W_{1}^{p}\left(R^{l}, d^{l} x\right)$ of the quasi-linear partial differential system of elliptic type, establish certain a priori estimations of this solution and its derivatives (applying the definition of a weak solution and assuming that element $\vec{v} \in W_{1,0}^{q}\left(R^{l}, d^{l} x\right)$, we are obtaining the theorems about this solution); study the properties of some functions of this solution $\vec{u} \in W_{1}^{p}\left(R^{l}, d^{l} x\right)$ (in the simplest case $\psi(\vec{u})=-\ln 2(1-|\vec{u}|+\varepsilon)$ ).

Applying this definition of a weak solution, we compile the following differential form $h_{\lambda}^{p}: W_{1}^{p} \times W_{1}^{q} \rightarrow R$ as

$$
h_{\lambda}^{p}(\vec{u}, \vec{\nu}) \equiv \lambda\langle\vec{u}, \vec{\nu}\rangle+\langle\nabla \vec{\nu} \circ a \circ \nabla \vec{u}\rangle+\langle\vec{b}(x, \vec{u}, \nabla \vec{u}), \vec{\nu}\rangle
$$

which is well defined over the functional space $W_{1}^{p}\left(R^{l}, d^{l} x\right) \times W_{1}^{q}\left(R^{l}, d^{l} x\right)$.

Let us assume that function $\vec{u} \in W_{1}^{p}\left(R^{l}, d^{l} x\right)$ is the solution of (1) that means that for an arbitrary function $\vec{v} \in W_{1,0}^{q}\left(R^{l}, d^{l} x\right)$ holds an integral tautology

$$
h_{\lambda}^{p}(\vec{u}, \vec{\nu}) \equiv \lambda\langle\vec{u}, \vec{v}\rangle+\left\langle\sum_{i, j=1, \ldots, l} a_{i j} \frac{\partial}{\partial x_{j}} \vec{u}, \frac{\partial}{\partial x_{i}} \vec{v}\right\rangle+\langle\vec{b}, \vec{v}\rangle=0 .
$$

To prove that function $\vec{u}$ is Holder continuous let us introduce a function of the solution of (1) as

$$
w(x)=\psi(\vec{u})(x)=-\ln \left(\frac{\operatorname{osc}\left\{u, K_{2 R}\right\}-|\vec{u}(x)|+\varepsilon}{\delta_{2} \operatorname{osc}\left\{u, K_{2 R}\right\}}\right)
$$

then we are going to show that

$$
-\ln \left(\frac{\operatorname{osc}\left\{u, K_{2 R}\right\}-|\vec{u}(x)|+\varepsilon}{\delta_{2} \operatorname{osc}\left\{u, K_{2 R}\right\}}\right) \leq M
$$

and

$$
\frac{\delta_{2} \operatorname{osc}\left\{u, K_{2 R}\right\}}{\operatorname{osc}\left\{u, K_{2 R}\right\}-|\vec{u}(x)|+\varepsilon} \leq \exp (M)
$$


and its conclusion

$$
|\vec{u}(x)| \leq\left(1-\exp (-M) \delta_{2}\right) \operatorname{osc}\left\{u, K_{2 R}\right\}+\varepsilon,
$$

where $u=|\vec{u}|$

Let us assume that in the integral tautology of weak solution $\vec{u} \in$ $W_{1}^{p}\left(R^{l}, d^{l} x\right)$ the function $v \in W_{1,0}^{q}\left(R^{l}, d^{l} x\right)$ is $\vec{u}|\vec{u}|^{p-1}$ then we obtain

$$
\lambda\left\langle\vec{u}, \vec{u}|\vec{u}|^{p-1}\right\rangle+\left\langle\sum_{i, j=1, \ldots l} a_{i j} \frac{\partial}{\partial x_{j}} \vec{u}, \frac{\partial}{\partial x_{i}}\left(\vec{u}|\vec{u}|^{p-1}\right)\right\rangle+\left\langle\vec{b}, \vec{u}|\vec{u}|^{p-1}\right\rangle=0,
$$

and

$\lambda\|\vec{u}\|^{p}+\frac{4(p-1)}{p^{2}}\left\langle\sum_{i, j=1, \ldots l} a_{i j} \frac{p}{2}|\vec{u}|^{\frac{p-2}{2}} \nabla_{j} \vec{u}, \frac{p}{2}|\vec{u}|^{\frac{p-2}{2}} \nabla_{i} \vec{u}\right\rangle+\left\langle\vec{b}, \vec{u}|\vec{u}|^{p-1}\right\rangle=0$,

let denote $\vec{w}=\vec{u}|\vec{u}|^{\frac{p-2}{2}}$ and respectively $\nabla \vec{w}=\frac{p}{2}|\vec{u}|^{\frac{p-2}{2}} \nabla \vec{u}$, the in all $R^{l}$, applying Holder and Young inequalities to Lebesgue's norms, we have

$$
\begin{aligned}
& \left|\left\langle\vec{b}, \vec{u}|\vec{u}|^{p-1}\right\rangle\right| \leq\left(\left(\frac{\varepsilon^{2}}{p}+1\right) c(\beta)+\frac{1}{\sigma^{q} q}\right)\|\vec{w}\|^{2}+ \\
& +\left(\frac{\beta \varepsilon^{2}}{p}+\beta+\frac{1}{p} \frac{1}{\varepsilon^{2}}\right)\langle\nabla \vec{w} \circ a \circ \nabla \vec{w}\rangle+\frac{\sigma^{p}}{p}\left\|\mu_{3}\right\|^{p},
\end{aligned}
$$

or

$$
\begin{aligned}
& \lambda\|\vec{u}\|^{p}+\frac{4(p-1)}{p^{2}}\langle\nabla \vec{w} \circ a \circ \nabla \vec{w}\rangle \leq\left(\left(\frac{\varepsilon^{2}}{p}+1\right) c(\beta)+\frac{1}{\sigma^{q} q}\right)\|\vec{w}\|^{2}+ \\
& +\left(\frac{\beta \varepsilon^{2}}{p}+\beta+\frac{1}{p} \frac{1}{\varepsilon^{2}}\right)\langle\nabla \vec{w} \circ a \circ \nabla \vec{w}\rangle+\frac{\sigma^{p}}{p}\left\|\mu_{3}\right\|^{p} .
\end{aligned}
$$

Let $K_{\rho_{0}}, K_{\rho}$ and $K_{b \rho}$ be concentric balls and constant $b$ such that $b \rho \leq \rho_{0}, b>1, c_{1} \leq 1, \delta \leq 1, \vartheta<1$ and $\Omega_{\rho}=\Omega \cap K_{\rho}$. The $\varsigma(x)$ is a cutoff function in the ball $K_{2 R}$ and let us choose $v=\varsigma^{p} \vec{u}|\vec{u}|^{p-2}$, we have

$$
\begin{aligned}
\lambda\left\langle\vec{u}, \varsigma^{p} \vec{u}|u|^{p-2}\right\rangle_{K_{2 R}} & +\left|\left\langle\sum_{i, j=1, \ldots l} a_{i j} \frac{\partial}{\partial x_{j}} \vec{u}, \frac{\partial}{\partial x_{i}}\left(\varsigma^{p} \vec{u}|\vec{u}|^{p-2}\right)\right\rangle_{K_{2 R}}\right| \leq \\
& \leq\left|\left\langle\vec{b}, \varsigma^{p} \vec{u}|\vec{u}|^{p-1}\right\rangle_{K_{2 R}}\right|, \\
& \left\langle\sum_{i, j=1, \ldots l} a_{i j} \frac{\partial}{\partial x_{j}} \vec{u}, \frac{\partial}{\partial x_{i}}\left(\varsigma^{p} \vec{u}|\vec{u}|^{p-2}\right)\right\rangle_{K_{2 R}}= \\
& =(p-1)\left\langle\sum_{i, j=1, \ldots l} \varsigma^{p} a_{i j}|\vec{u}|^{\frac{p-2}{2}} \frac{\partial}{\partial x_{j}} \vec{u},|\vec{u}|^{\frac{p-2}{2}} \frac{\partial}{\partial x_{i}} \vec{u}\right\rangle_{K_{2 R}}+ \\
& +p\left\langle\sum_{i, j=1, \ldots l} a_{i j} \vec{u}|\vec{u}|^{p-2} \varsigma^{p-1} \frac{\partial}{\partial x_{j}} \vec{u}, \frac{\partial}{\partial x_{i}} \varsigma\right\rangle_{K_{2 R}}
\end{aligned}
$$


we denote $\vec{w}=\vec{u}|\vec{u}|^{\frac{p-2}{2}}$ then

$$
\left\langle\sum_{i, j=1, \ldots, l} \varsigma^{p} a_{i j} \frac{\partial}{\partial x_{j}} \vec{u}, \frac{\partial}{\partial x_{i}}\left(\vec{u}|\vec{u}|^{p-2}\right)\right\rangle_{K_{2 R}}=\frac{4(p-1)}{p^{2}}\left\langle\varsigma^{p} \nabla \vec{w} \circ a \circ \nabla \vec{w}\right\rangle_{K_{2 R}},
$$

after the transformation of the second term on the right side

$$
\left\langle\sum_{i, j=1, \ldots l} a_{i j} \vec{u}|\vec{u}|^{p-2} \frac{\partial}{\partial x_{j}} \vec{u}, \frac{\partial}{\partial x_{i}}\left(\varsigma^{p}\right)\right\rangle_{K_{2 R}}=p\left\langle\sum_{i, j=1, \ldots l} \varsigma^{p-1} a_{i j} \vec{u}|\vec{u}|^{p-2} \frac{\partial}{\partial x_{j}} \vec{u}, \frac{\partial}{\partial x_{i}} \varsigma\right\rangle_{K_{2 R}}
$$

by Young inequality, we have had

$$
\begin{aligned}
& \left\langle\sum_{i, j=1, \ldots, l} \varsigma^{p-1} a_{i j} \vec{u}|\vec{u}|^{p-2} \frac{\partial}{\partial x_{j}} \vec{u}, \frac{\partial}{\partial x_{i}} \varsigma\right\rangle_{K_{2 R}} \leq \\
& \leq \frac{1}{p}\left\langle\sum_{i, j=1, \ldots, l} \varsigma^{p-1} a_{i j} \vec{u}|\vec{u}|^{p-2}\left|\frac{\partial}{\partial x_{j}} \vec{u}\right|^{p},\left|\frac{\partial}{\partial x_{i}} \varsigma\right|^{p}\right\rangle_{K_{2 R}}+ \\
& +\frac{1}{q}\left\langle\sum_{i, j=1, \ldots, l} \varsigma^{p-1} a_{i j} \vec{u}|\vec{u}|^{p-2} \frac{\partial}{\partial x_{j}} \vec{u}, \frac{\partial}{\partial x_{i}} \varsigma\right\rangle_{K_{2 R}} .
\end{aligned}
$$

Theorem 2. Let $\delta_{2}, \delta_{3}$ be positive constants such that

$\operatorname{mes}\left\{x \in K_{R},|\vec{u}(x)| \leq \max _{K_{2 R}}|\vec{u}(x)|-\delta_{2} \operatorname{osc}\left\{u, K_{2 R}\right\}\right\} \geq\left(1-\delta_{3}\right) \operatorname{mes}_{R}$,

then there is a positive constant $\delta_{1}$ that depends only on the ellipticity, smoothness of coefficients, the dimension of space, and constants $\delta_{2}, \delta_{3}$ such that

$$
\operatorname{osc}\left\{u, K_{R}\right\} \leq \max _{K_{R}}|u(x)| \leq\left(1-\delta_{1}\right) \operatorname{osc}\left\{u, K_{2 R}\right\}+R^{1-\frac{l}{m}}
$$

for all $m>l$, where $u=|\vec{u}|$

Comment. From Relliha - Kondrashov theorem for Sobolev space and statement that all elements belonging Sobolev space $W_{1}^{p}\left(R^{l}, d^{l} x\right), l<p \leq$ $\infty$ belong to the Holder continuous functional space of $\alpha=\frac{p-l}{p}$ degree we can conclude it is enough to consider only a case when $p \leq l$.

Proof. For a positive number $\delta_{2}$, let us consider the function

$$
w(x)=\psi(\vec{u})(x)=-\ln \left(\frac{\operatorname{osc}\left\{u, K_{2 R}\right\}-|\vec{u}(x)|+\varepsilon}{\delta_{2} \operatorname{osc}\left\{u, K_{2 R}\right\}}\right)
$$


and let us assume that in the ball $K_{R}$ the estimation

$$
-\ln \left(\frac{\operatorname{osc}\left\{u, K_{2 R}\right\}-|\vec{u}(x)|+\varepsilon}{\delta_{2} \operatorname{osc}\left\{u, K_{2 R}\right\}}\right)<L
$$

holds then we have the estimation

$$
\delta_{2} \operatorname{osc}\left\{u, K_{2 R}\right\} \exp (-L)<\operatorname{osc}\left\{u, K_{2 R}\right\}-|\vec{u}(x)|+\varepsilon
$$

or

$|\vec{u}(x)|<\operatorname{osc}\left\{u, K_{2 R}\right\}-\delta_{2} \operatorname{osc}\left\{u, K_{2 R}\right\} \exp (-L)+\varepsilon=\left(1-\delta_{2} \exp (-L)\right) \operatorname{osc}\left\{u, K_{2 R}\right\}+\varepsilon$

put $\delta_{1}=\delta_{2} \exp (-L)$, we obtain

$$
\operatorname{osc}\left\{u, K_{R}\right\} \leq \max _{K_{R}}|\vec{u}(x)| \leq\left(1-\delta_{1}\right) \operatorname{osc}\left\{u, K_{2 R}\right\}+R^{1-\frac{l}{m}},
$$

where $\varepsilon=R^{1-\frac{l}{m}}$. Theorem 2 has been proven.

Assuming $\vec{v}=\vec{u}|\vec{u}|^{p-2}$ in

$$
\lambda\langle\vec{u}, \vec{v}\rangle+\left\langle\sum_{i, j=1, \ldots, l} a_{i j} \frac{\partial}{\partial x_{j}} \vec{u}, \frac{\partial}{\partial x_{i}} \vec{v}\right\rangle+\langle\vec{b}, \vec{v}\rangle=0
$$

denoting $\vec{w}=\vec{u}|\vec{u}|^{\frac{p-2}{2}}$ and $\nabla \vec{w}=\frac{p}{2}|\vec{u}|^{\frac{p-2}{2}} \nabla \vec{u}$, we are obtaining

$$
\begin{aligned}
& \lambda\|\vec{u}\|^{p}+\frac{4(p-1)}{p^{2}}\left\langle\nabla\left(\vec{u}|\vec{u}|^{\frac{p-2}{2}}\right) \circ a \circ \nabla\left(\vec{u}|\vec{u}|^{\frac{p-2}{2}}\right)\right\rangle \leq\left(\left(\frac{\varepsilon^{2}}{p}+1\right) c(\beta)+\frac{1}{\sigma^{q} q}\right)\|\vec{u}\|^{p}+ \\
& +\left(\frac{\beta \varepsilon^{2}}{p}+\beta+\frac{1}{p} \frac{1}{\varepsilon^{2}}\right)\left\langle\nabla\left(\vec{u}|\vec{u}|^{\frac{p-2}{2}}\right) \circ a \circ \nabla\left(\vec{u}|\vec{u}|^{\frac{p-2}{2}}\right)\right\rangle+\frac{\sigma^{p}}{p}\left\|\mu_{3}\right\|^{p} .
\end{aligned}
$$

Let assume

$$
v(x)=\frac{\vec{\xi}(x)}{\operatorname{osc}\left\{u, K_{2 R}\right\}-|\vec{u}(x)|+\varepsilon} \equiv \frac{\vec{\xi}}{P},
$$

where the $\vec{\xi}$ countable smooth cutoff in the ball $K_{2 R}$ and notice that can be written

$$
\nabla \vec{w}=\frac{\nabla \vec{u}}{o s c\left\{u, K_{2 R}\right\}-|\vec{u}|+\varepsilon},
$$

then we have

$$
\lambda\left\langle\vec{u}, \frac{\vec{\xi}}{P}\right\rangle+\left\langle\sum_{i, j=1, \ldots l} a_{i j} \frac{\partial}{\partial x_{j}} \vec{u}, \frac{\partial}{\partial x_{i}} \frac{\vec{\xi}}{P}\right\rangle+\left\langle\vec{b}, \frac{\vec{\xi}}{P}\right\rangle=0
$$


and

$$
\begin{aligned}
& \left\langle\vec{b}, \frac{\vec{\xi}}{P}\right\rangle \leq\left\langle\mu_{1}|\nabla \vec{u}|+\mu_{2}|\vec{u}|+\mu_{3}, \frac{|\vec{\xi}|}{P}\right\rangle= \\
& =\left\langle\mu_{1}|\nabla \vec{u}|, \frac{|\vec{\xi}|}{P}\right\rangle+\left\langle+\mu_{2}|\vec{u}|, \frac{|\vec{\xi}|}{P}\right\rangle+\left\langle\mu_{3}, \frac{|\vec{\xi}|}{P}\right\rangle
\end{aligned}
$$

cutoff in the ball $K_{2 R}$ by integrating over the ball $K_{2 R}$, we have

$$
\begin{aligned}
& \left\langle\vec{b}, \frac{\vec{\xi}}{P}\right\rangle_{K_{2 R}} \leq\left\langle\mu_{1}|\nabla \vec{u}|+\mu_{2}|\vec{u}|+\mu_{3}, \frac{|\vec{\xi}|}{P}\right\rangle_{K_{2 R}}= \\
& =\left\langle\mu_{1}|\nabla \vec{u}|, \frac{|\vec{\xi}|}{P}\right\rangle_{K_{2 R}}+\left\langle\mu_{2}|\vec{u}|, \frac{|\vec{\xi}|}{P}\right\rangle_{K_{2 R}}+\left\langle\mu_{3}, \frac{|\vec{\xi}|}{P}\right\rangle_{K_{2 R}},
\end{aligned}
$$

by Holder estimation

$$
\left\langle\mu_{1}|\nabla \vec{u}|, \frac{|\vec{\xi}|}{P}\right\rangle_{K_{2 R}}=\left\langle\mu_{1}|\nabla \vec{w}|,|\vec{\xi}|\right\rangle_{K_{2 R}} \leq\|\nabla \vec{w}\|_{K_{2 R}}\left\|\mu_{1}|\vec{\xi}|\right\|_{K_{2 R}},
$$

and Young inequality

$$
\|\nabla \vec{w}\|_{K_{2 R}}\left\|\mu_{1}|\vec{\xi}|\right\|_{K_{2 R}} \leq\|\nabla \vec{w}\|_{K_{2 R}}^{2}+\left\|\mu_{1}|\vec{\xi}|\right\|_{K_{2 R}}^{2},
$$

from form-boundary, we are obtaining

$$
\left\|\mu_{1}|\vec{\xi}|\right\|_{K_{2 R}}^{2} \leq\left\langle\mu_{1}^{2}|\vec{\xi}|^{2}\right\rangle_{K_{2 R}} \leq \beta\|\Delta \vec{\xi}\|_{K_{2 R}}^{2}+c(\beta)\|\nabla \vec{\xi}\|_{K_{2 R}}^{2},
$$

a similar consideration gives us

$$
\left\langle\mu_{2}|\vec{u}|, \frac{|\vec{\xi}|}{P}\right\rangle_{K_{2 R}} \leq\|\vec{u}\|_{K_{2 R}}\left\|\mu_{2} \frac{|\vec{\xi}|}{P}\right\|_{K_{2 R}}
$$

Since $\mu_{2}^{2} \in P K_{\beta}(A)$ we are applying form-boundary and obtaining

$$
\left\|\mu_{2} \frac{|\vec{\xi}|}{P}\right\|_{K_{2 R}}^{2} \leq \varepsilon^{-2}\left(\beta\|\Delta \vec{\xi}\|_{K_{2 R}}^{2}+c(\beta)\|\nabla \vec{\xi}\|_{K_{2 R}}^{2}\right) .
$$

If we assume that $\mu_{1} \in L^{\infty}\left(K_{2 R}\right)$ we have an estimation

$$
\left\|\mu_{2} \frac{|\vec{\xi}|}{P}\right\|_{K_{2 R}} \leq \varepsilon^{-1} \text { vrai } \max \left(\mu_{2}|\vec{\xi}|\right)\left(\text { mes }_{2 R}\right)^{\frac{1}{2}}
$$


the assumption $\mu_{1} \in L^{\infty}\left(K_{2 R}\right)$ imposes too strong conditions on the coefficients.

After reducing, we are obtaining that the function

$$
\vec{v}(x)=\frac{\vec{\xi}(x)}{\operatorname{osc}\left\{u, K_{2 R}\right\}-|\vec{u}(x)|+\varepsilon} \equiv \frac{\vec{\xi}}{P}
$$

is bounded. Theorem 1 has been proved.

\section{References}

[1] D. R. Adams and L. I. Hedberg. Function Spaces and Potential Theory. Berlin: Springer-Verlag, 1996.

[2] S. Agmon, A. Douglis, and L. Nirenberg, "Estimates near the boundary for solutions of elliptic partial differential equations satisfying general boundary conditions I, II", Communications on pure and applied mathematics, vol. 12, 1959-1964.

[3] A. G. Berlyiand and Yu. A. Semenov, "On the Lp-theory of Schrodinger semigroups", Siberian mathematical journal, vol. 31, pp. 16-26, 1990.

[4] H. Brezis and A. Pazy, "Semigroups of nonlinear contractions on convex sets", Journal of functional analysis, vol. 6, no. 2, pp. 237-281, 1970. doi: 10.1016/ 0022-1236(70)90060-1

[5] F. E. Browder, "Existence of periodic solutions for nonlinear equations of evolution", Proceedings of the National Academy of sciences, vol. 53, no. 5, pp. 1100-1103, 1965. doi: 10.1073/ pnas.53.5.1100

[6] F. E. Browder, "Nonlinear equations of evolution and nonlinear accretive operators in Banach spaces", Bulletin of the American Mathematical Society, vol. 73, no. 6, pp. 867-874, 1967. doi: 10.1090/s0002-9904-1967-11820-2

[7] M. G. Crandall and A. Pazy, "Semi-groups of nonlinear contractions and dissipative sets", Journal of functional analysis, vol. 3, no. 3, pp. 376-418, 1969. doi: 10.1016/ 0022-1236(69)90032-9

[8] J.-M. Bony, "Calcul symbolique et propagation des singularités pour les équations aux Dérivées partielles non linéaires", Annales scientifiques de l'École normale supérieure, vol. 14, no. 2, pp. 209-246, 1981. doi: 10.24033/ asens.1404 
[9] H. Brezis, "Some variational problems with lack of compactness", in Nonlinear functional analysis and its applications, F. Browder, Ed. Providence: AMS, pp. 165-202, 1986.

[10] H. Brezis and J. M. Coron, "Convergence of solutions of H-systems or how to blow bubbles", Archive for rational mechanics and analysis, vol. 89, no. 1, pp. 21-56, 1985. doi: 10.1007/ bf00281744

[11] H. Brezis, J.-M. Coron, and E. H. Lieb, "Harmonic maps with defects", Communications in mathematical physics, vol. 107, no. 4, pp. 649-705, 1986. doi: $10.1007 /$ bf01205490

[12] H. Brezis and L. Nirenberg, "Positive solutions of nonlinear elliptic equations involving critical sobolev exponents", Communications on pure and applied mathematics, vol. 36, no. 4, pp. 437-477, 1983. doi: 10.1002/ cpa.3160360405

[13] F. Browder, "Fixed point theory and nonlinear problems", Bulletin of the american mathematical society, vol. 9, pp. 1-39, 1983.

[14] F. E. Browder and W. V. Petryshyn, "Approximation methods and the generalized topological degree for nonlinear mappings in Banach spaces", Journal of functional analysis, vol. 3, no. 2, pp. 217-245, 1969. doi: 10.1016/ 0022-1236(69)90041-x

[15] L. Caffarelli, R. Kohn, and L. Nirenberg, "Partial regularity of suitable weak solutions of the navier-stokes equations", Communications on pure and applied mathematics, vol. 35, no. 6, pp. 771-831, 1982. doi: 10.1002/ cpa.3160350604

[16] L. Caffarelli, L. Nirenberg, and J. Spruck, "The Dirichlet problem for nonlinear second-order elliptic equations I. Monge-Ampégre equation", Communications on pure and applied mathematics, vol. 37, no. 3, pp. 369-402, 1984. doi: 10.1002/ cpa.3160370306

[17] M. G. Crandall and P.-L. Lions, "Viscosity solutions of Hamilton-Jacobi equations", Transactions of the american mathematical society, vol. 277, no. 1, pp. 1-42, 1983. doi: 10.1090/ s0002-9947-1983-0690039-8

[18] B. Dacorogna, Weak Continuity and weak lower semi-continuity of nonlinear functionals, Berlin: Springer-Verlag, 1987.

[19] E. B. Davies, One parameter semigroups, San Diego: Academic Press, 1980.

[20] E. B. Davies, "Lp Spectral Theory of Higher-Order Elliptic Differential Operators", Bulletin of the London mathematical society, vol. 29, no. 5, pp. 513-546, 1997. doi: 10.1112/s002460939700324x

[21] E. DeGiorgi, "Sulla differenziabilita e l'analiticita delle estremali degli integrali multipli regolari", Memorie della Reale accademia delle scienze di Torino, Classe di Scienze Fisiche, Matematiche e Naturali., vol. 3, pp. 25-43, 1957. 
[22] E. DeGiorgi, "Un esempio di estremali discontinue per un problema variazionale di tipo ellitico", Bollettino della Unione Matematica Italiana. Series IV, no. 1, pp. 135-137, 1968.

[23] R. J. DiPerna, "Compensated compactness and general systems of conservation laws", Transactions of the American mathematical society, vol. 292, no. 2, pp. 383-420, 1985. doi: 10.1090/s0002-99471985-0808729-4

[24] R. J. DiPerna and P. L. Lions, "On the cauchy problem for Boltzmann equations: Global existence and weak stability", The annals of mathematics, vol. 130, no. 2, p. 321, 1989. doi: 10.2307/ 1971423

[25] R. J. DiPerna and A. Majda, "Reduced hausdorff dimension and concentration-cancellation for two-dimensional incompressible flow", Journal of the American mathematical society, vol. 1, no. 1, pp. 59-95, 1988. doi: 10.1090/ s0894-0347-1988-0924702-6

[26] L. C. Evans, Weak convergence methods for nonlinear partial differential equations. Providence: AMS, 1988.

[27] L. Giacomelli and H. Knüpfer, "A free boundary problem of fourth order: Classical solutions in Weighted Hölder spaces", Communications in partial differential equations, vol. 35, no. 11, pp. 2059-2091, 2010. doi: 10.1080/ 03605302.2010.494262

[28] M. Giaquinta, Multiple integrals in the calculus of variations and nonlinear elliptic systems. Princeton: Princeton University Press, 1983.

[29] M. Giaquinta, Introduction to Regularity Theory for Nonlinear Elliptic Systems. Basel: Birkhauser, 1993.

[30] B. Gidas, W.-M. Ni, and L. Nirenberg, "Symmetry and related properties via the maximum principle", Communications in mathematical physics, vol. 6, pp. 883-901, 1981.

[31] D. Gilbarg and N. S. Trudinger, Elliptic partial differential equations of second order, 2nd ed., Berlin: Springer, 1983.

[32] E. Giusti, Minimal Surfaces and Functions of Bounded Variation. Basel: Birkhauser, 1984.

[33] R. Landes and V. Mustonen, "On pseudo-monotone operators and nonlinear noncoercive variational problems on unbounded domains", Mathematische annalen, vol. 248, no. 3, pp. 241-246, 1980. doi: $10.1007 /$ bf01420527 
[34] G. J. Minty, "Monotone (nonlinear) operators in Hilbert Space", Duke mathematical journal, vol. 29, no. 3, pp. 341-346, 1962. doi: 10.1215/ s0012-7094-62-02933-2

[35] G. J. Minty, "On the generalization of a direct method of the calculus of variations", Bulletin of the American mathematical society, vol. 73, no. 3, pp. 315-321, 1967. doi: 10.1090/ s0002-9904-1967-11732-4

[36] I. Miyadera, "On perturbation theory for semi-groups of operators", Tohoku mathematical journal, vol. 18, no. 3, pp. 299-310, 1966. doi: $10.2748 / \mathrm{tmj} / 1178243419$

[37] J. Necas, Introduction to the theory of nonlinear elliptic equations, New York: Teubner and Wiley, 1983.

[38] E. V. Radkevich, "Equations with nonnegative characteristic form. I", Journal of mathematical sciences, vol. 158, no. 3, pp. 297-452, 2009. doi: 10.1007/ s10958-009-9394-2

[39] E. Stein, Harmonic analysis: real variable methods, orthogonality, and oscillatory integrals, Princeton: Princeton University. Press, 1993.

[40] M. Struwe, "A global compactness result for elliptic boundary value problems involving limiting nonlinearities", Mathematische zeitschrift, vol. 187, no. 4, pp. 511-517, 1984. doi: 10.1007/ bf01174186

[41] J. Sylvester and G. Uhlmann, "A global uniqueness theorem for an inverse boundary value problem", The annals of mathematics, vol. 125, no. 1, pp. 153-169, 1987. doi: 10.2307/ 1971291

[42] M. Taylor, Partial differential equations, 3 vols. Berlin: Springer, 1996.

[43] L. Veron, Singularities of solutions of second order quasilinear equations, New York: Longman, 1996.

[44] F. B. Weissler, "Single point blow-up for a semilinear initial value problem", Journal of differential equations, vol. 55, no. 2, pp. 204-224, 1984. doi: 10.1016/ 0022-0396(84)90081-0

[45] M. I. Yaremenko, "The existence of a solution of evolution and elliptic equations with singular coefficients", Asian journal of mathematics and computer research, vol. 15, no. 3, pp. 172-204, 2017.

[46] M. I. Yaremenko, "Quasi-linear evolution and elliptic equations", Journal of progressive research in mathematics, vol. 11, 13 pp. 1645-1669, 2017. 
[47] M. I. Yaremenko, "The sequence of semigroups of nonlinear operators and their applications to study the Cauchy problem for parabolic equations", Scientific journal of the ternopil national technical university, vol. 14, pp. 149-160, 2016.

\section{Mykola I. Yaremenko}

National Technical University of Ukraine

"Igor Sikorsky Kyiv Polytechnic Institute" Kyiv, Ukraine

e-mail: math.kiev@gmail.com 